\title{
Effect of Nusinersen on Respiratory Muscle Function in Different Subtypes of Type 1 Spinal Muscular Atrophy
}

Antonella LoMauro ${ }^{1}$; Chiara Mastella ${ }^{2}$; Katia Alberti2; Riccardo Masson ${ }^{3}$; Andrea Aliverti $^{1 *}$; Giovanni Baranello ${ }^{3,4 *}$

\footnotetext{
${ }^{1}$ Dipartimento di Elettronica, Informazione e Bioingegneria, Politecnico di Milano, Piazza Leonardo Da Vinci, Milan, Italy

2 Fondazione IRCCS Cà' Granda Ospedale Maggiore Policlinico, SAPRE-UONPIA, Neuropsichiatria dell'Infanzia e dell'Adolescenza, Milan, Italy

${ }^{3}$ UO Neurologia dello Sviluppo, Fondazione IRCCS Istituto Neurologico Carlo Besta, Milan, Italy

4 Dubowitz Neuromuscular Centre, NIHR Great Ormond Street Hospital Biomedical Research Centre, UCL Great Ormond Street Institute of Child Health London, UK

* Equal contribution
}

Correspondence: antonella.lomauro@polimi.it 
To the editor

Spinal muscular atrophy (SMA) is the leading inherited cause of infant death. However, the rapidly evolving landscape of therapeutic options is dramatically changing the natural history of the disease. Nusinersen was the first drug approved for the treatment of SMA, and it is now commercially available in many countries worldwide. Very recently, the gene therapy onasemnogene abeparvovec-xioi has also received approval from FDA for the treatment of patients younger than 2 years of age. Nusinersen is administered intrathecally and consists of an antisense oligonucleotide designed to modify pre-messenger RNA thus increasing the level of SMN protein(1). A phase 3 randomized, double-blind, sham-controlled, clinical trial in patients with SMA1, the most severe form of SMA, showed that patients treated with nusinersen had a significant motor milestone response and a higher likelihood of event-free survival ${ }^{2}$ (i.e. free from tracheostomy or non-invasive permanent ventilation). However, there is no information on the effects of the treatment on respiratory function that strongly affects the prognosis in SMA1, as respiratory problems are the major cause of hospitalization, morbidity and mortality. Ribcage muscle weakness is a distinctive feature of SMA1 since infancy, while the diaphragm is spared and leads the breathing function(3); and natural history studies $(4,5)$ showed that the majority of surviving SMA1 children become ventilator dependent. The assessment of respiratory function in infants is challenging because it usually requires invasive and/or volitional tests(6). We have previously reported how feasible and informative the non-invasive measurement of respiratory pattern during quiet breathing by optoelectronic plethysmography can be, even in uncooperative infants. We have characterized the specific ventilatory and thoraco-abdominal patterns during quiet breathing of infants and children affected by the three forms of SMA. Specifically, SMA1 patients were characterized by rapid and shallow breathing with paradoxical thoraco-abdominal motion(3) and bell-shaped thorax(7). The study was 
carried out in 32 untreated SMA 1 patients, who might be considered a control group of natural history patients. In the present study, respiratory function data from the control group was compared with that of a new cohort of 27 SMA1 nusinersen treated infants and children. For the very first time we were able to understand if and how nusinersen impacts on the respiratory function in the most severe form of the disease.

In order to better capture possible response to treatment, which has been reported to be variable depending on different factors including age at treatment, disease duration and severity, we decided to divide both the control and the treated SMA1 patients into the 3 subtypes, defined according to age of symptoms onset and clinical presentation (symptoms in the first 2 weeks of life: 1A; within the third month: $1 \mathrm{~B}$; between 3 and 6 months: 1C)(8).

Regrouping our cohort of natural history according to the subtypes (17 A-B patients: $\mathrm{AB}_{\mathrm{NH}}$ and $15 \mathrm{C}: \mathrm{C}_{\mathrm{NH}}$ ), differences emerged in the ventilatory pattern (Figure1). While total minute ventilation was similar, its two components, namely respiratory rate and tidal volume differed, resulting in more severe rapid and shallow breathing index (RSBi) in $\mathrm{AB}_{\mathrm{NH}}$ (Table1). In the treated group (15 A-B patients: $\mathrm{AB}_{\mathrm{TR}}$ and $12 \mathrm{C}: \mathrm{C}_{\mathrm{TR}}$ ), respiratory rate still remained higher in the $\mathrm{AB}_{\mathrm{TR}}$, but tidal volume, minute ventilation and $R S B i$ were similar between $A_{T R}$ and $C_{T R}$ (Table1).

In addition to motor function improvement, the most striking difference between the groups emerged in the action of ribcage muscles, as indicated by the percentage contribution of pulmonary ribcage to tidal volume $\left(\% \Delta \mathrm{V}_{\mathrm{RC}, \mathrm{P}}\right)(3)$ in Figure 1.

In untreated patients, $\% \Delta V_{R C, P}$ did not differ $(p=0.952)$ between $A B_{N H}$ and $C_{N H}$ being always negative, thus confirming the systematic presence of paradoxical inspiratory inward thoracic motion. By contrast in the treated groups, $A_{\mathrm{TR}}$ were similar to natural history $A B$ patients $(p=0.664)$, while $C_{T R}$ were characterized by the highest 
values of $\% \Delta V_{R C, P}(p<0.001)$ that were always positive, thus demonstrating absence of thoraco-abdominal breathing asynchrony. Importantly, $\mathrm{C}_{\mathrm{TR}}$ at baseline had clinical severity similar to the correspondent untreated group, as their motor function, assessed by the CHOP INTEND scale $(9,10)$, did not significantly differ $(p=0.21)$.

Two important considerations emerged from these results: nusinersen had an ameliorative effect also on the respiratory function of SMA1 infants and children; however, disease subtype seems to play a crucial role in the response to the drug. While respiratory pattern of treated SMA1 A-B patients did not significantly differ from untreated patients, SMA 1C patients were the best responders in terms of ventilatory and thoraco-abdominal patterns. Importantly, both age of starting and treatment duration were similar between $\mathrm{AB}_{\mathrm{TR}}$ and $\mathrm{C}_{\mathrm{TR}}$ (Table1) and therefore they did not affect results. The disappearance of paradoxical ribcage motion indicated that ribcage muscles became strong enough to counteract the negative pleural pressure swings developed by the contraction of the diaphragm. The main consequences of the improved action of respiratory muscles were the significantly reduced bellshaped chest index and daily hours of mechanical ventilation needed by $\mathrm{C}_{\mathrm{TR}}$ compared to $\mathrm{AB}_{\mathrm{TR}}$, whose values did not differ to natural history groups (Table1).

This is the first evidence of the efficacy of nusinersen on respiratory function in SMA1 patients and it did not only consider the use (or not) of permanent ventilation as respiratory outcome. However, the responsiveness to the drug was dramatically different according to disease subtypes, with $C_{T R}$ being the best responders; $A_{T R}$ showed significant improvement in motor function but respiratory function almost followed natural history pattern even after at least 1 year of treatment. With the exception of one child undergoing tracheostomy after 18 months of treatment, none of the other patients either increased number of hours of ventilation or became ventilator-dependent. These findings should be confirmed in longitudinal studies 
tracking the effects of nusinersen with growth. Interestingly, SMN2 gene copy number was significantly higher in the treated group ( $50 \%$ of $\mathrm{C}_{\mathrm{TR}}$ with 3 copies), thus suggesting a possible role of this factor in influencing pharmacodynamics and thus response to treatment, particularly considering respiratory function. However, this finding needs further investigation, as the actual role of SMN2 gene copy number in influencing clinical and motor response is still controversial $(11,12)$.

Although nusinersen has opened up scenarios of motivated hope, our data further highlighted that adequate supportive care should remain imperative for SMA1 infants and children, particularly subtypes 1A-B, but also 1C. Albeit an increasing number of patients can now benefit from the approved treatments or from other drugs under clinical development, in many cases SMA still remains a severe neuromuscular disorder in which respiratory and bulbar involvement is required to be properly managed according to the standard of care recommendations for SMA(13). Finally, the different effect of systemic versus intrathecally delivered drugs on respiratory function in SMA1 patients could be tested in the near future.

The study was approved by the Research Ethics Board of the Carlo Besta Neurological Research Institute (registration number: CE: 20/2014) and all parents signed a written informed consent.

Some of the results of this study have been previously reported in the form of an abstract and they will be presented as poster presentation at the $24^{\text {th }}$ International Annual Congress of the World Muscle Society (Copenhagen, $1^{\text {st }} 5^{\text {th }}$ October 2019 , (14)). 


\section{Bibliography}

1. Finkel RS, Chiriboga CA, Vajsar J, Day JW, Montes J, De Vivo DC, Yamashita M, Rigo F, Hung G, Schneider E, Norris DA, Xia S, Bennett CF, Bishop KM. Treatment of infantile-onset spinal muscular atrophy with nusinersen: a phase 2, open-label, dose-escalation study. Lancet (London, England) 2016;388:3017-3026.

2. Finkel RS, Mercuri E, Darras BT, Connolly AM, Kuntz NL, Kirschner J, Chiriboga CA, Saito K, Servais L, Tizzano E, Topaloglu H, Tulinius M, Montes J, Glanzman AM, Bishop K, Zhong ZJ, Gheuens S, Bennett CF, Schneider E, Farwell W, De Vivo DC, ENDEAR Study Group. Nusinersen versus Sham Control in Infantile-Onset Spinal Muscular Atrophy. $N$ Engl J Med 2017;377:1723-1732.

3. LoMauro A, Aliverti A, Mastella C, Arnoldi MT, Banfi P, Baranello G. Spontaneous Breathing Pattern as Respiratory Functional Outcome in Children with Spinal Muscular Atrophy (SMA). In: Singh RN, editor. PLoS One 2016;11:e0165818.

4. Kolb SJ, Coffey CS, Yankey JW, Krosschell K, Arnold WD, Rutkove SB, Swoboda KJ, Reyna SP, Sakonju A, Darras BT, Shell R, Kuntz N, Castro D, Parsons J, Connolly AM, Chiriboga CA, McDonald C, Burnette WB, Werner K, Thangarajh M, Shieh PB, Finanger E, Cudkowicz ME, McGovern MM, McNeil DE, Finkel R, Iannaccone ST, Kaye E, Kingsley A, et al. Natural history of infantile-onset spinal muscular atrophy. Ann Neurol 2017;82:883-891.

5. Finkel RS, McDermott MP, Kaufmann P, Darras BT, Chung WK, Sproule DM, Kang PB, Foley AR, Yang ML, Martens WB, Oskoui M, Glanzman AM, Flickinger J, Montes J, Dunaway S, O’Hagen J, Quigley J, Riley S, Benton M, Ryan PA, Montgomery M, Marra J, Gooch C, De Vivo DC. Observational study of spinal muscular atrophy type I and implications for clinical trials. Neurology 2014;83:810-817.

6. Nicot F, Hart N, Forin V, Boulé M, Clément A, Polkey MI, Lofaso F, Fauroux B. Respiratory muscle testing: a valuable tool for children with neuromuscular disorders. Am J Respir Crit Care Med 2006;174:67-74.

7. LoMauro A, Banfi P, Mastella C, Alberti K, Baranello G, Aliverti A. A New Method for Measuring Bell-Shaped Chest Induced by Impaired Ribcage Muscles in Spinal Muscular Atrophy Children. Front Neurol 2018;9:703.

8. Finkel R, Bertini E, Muntoni F, Mercuri E, ENMC SMA Workshop Study 
Group. 209th ENMC International Workshop: Outcome Measures and Clinical Trial Readiness in Spinal Muscular Atrophy 7-9 November 2014, Heemskerk, The Netherlands. Neuromuscul Disord 2015;25:593-602.

9. Glanzman AM, Mazzone E, Main M, Pelliccioni M, Wood J, Swoboda KJ, Scott C, Pane M, Messina S, Bertini E, Mercuri E, Finkel RS. The Children's Hospital of Philadelphia Infant Test of Neuromuscular Disorders (CHOP INTEND): test development and reliability. Neuromuscul Disord 2010;20:15561.

10. Glanzman AM, McDermott MP, Montes J, Martens WB, Flickinger J, Riley S, Quigley J, Dunaway S, O’Hagen J, Deng L, Chung WK, Tawil R, Darras BT, Yang M, Sproule D, De Vivo DC, Kaufmann P, Finkel RS. Validation of the Children's Hospital of Philadelphia Infant Test of Neuromuscular Disorders (CHOP INTEND). Pediatr Phys Ther 2011;23:322-6.

11. Pane M, Coratti G, Sansone VA, Messina S, Bruno C, Catteruccia M, Sframeli M, Albamonte E, Pedemonte M, D’Amico A, Bravetti C, Berti B, Brigati G, Tacchetti P, Salmin F, de Sanctis R, Lucibello S, Piastra M, Genovese O, Bertini E, Vita G, Tiziano FD, Mercuri E, Italian Expanded Access Program Working Group. Nusinersen in type 1 spinal muscular atrophy: Twelve-month real-world data. Ann Neurol 2019;ana.25533.doi:10.1002/ana.25533.

12. Aragon-Gawinska K, Seferian AM, Daron A, Gargaun E, Vuillerot C, Cances C, Ropars J, Chouchane M, Cuppen I, Hughes I, Illingworth M, MariniBettolo C, Rambaud J, Taytard J, Annoussamy M, Scoto M, Gidaro T, Servais L. Nusinersen in patients older than 7 months with spinal muscular atrophy type 1. Neurology 2018;91:e1312-e1318.

13. Finkel RS, Mercuri E, Meyer OH, Simonds AK, Schroth MK, Graham RJ, Kirschner J, Iannaccone ST, Crawford TO, Woods S, Muntoni F, Wirth B, Montes J, Main M, Mazzone ES, Vitale M, Snyder B, Quijano-Roy S, Bertini E, Davis RH, Qian Y, Sejersen T, SMA Care group. Diagnosis and management of spinal muscular atrophy: Part 2: Pulmonary and acute care; medications, supplements and immunizations; other organ systems; and ethics. Neuromuscul Disord 2018;28:197-207.

14. LoMauro A, Masson R, Mastella C, Aliverti A, Baranello G. "The beneficial effect of nusinersen on the breathing pattern of SMA type 1 children.". Unpublished abstract, $2^{\text {nd }}$ April 2019, typescript. 


\section{Figure legend}

\section{Figure 1}

Median (symbols) and interquartile range (whiskers) of age (x-axis) and percentage contribution of pulmonary ribcage to tidal volume (y-axis) in SMA1 subtypes A-B (light grey), C (black) natural history children (square), and children treated with Nusinersen (circle).

Negative values indicate the presence of paradoxical inspiratory inward motion of the pulmonary ribcage. 


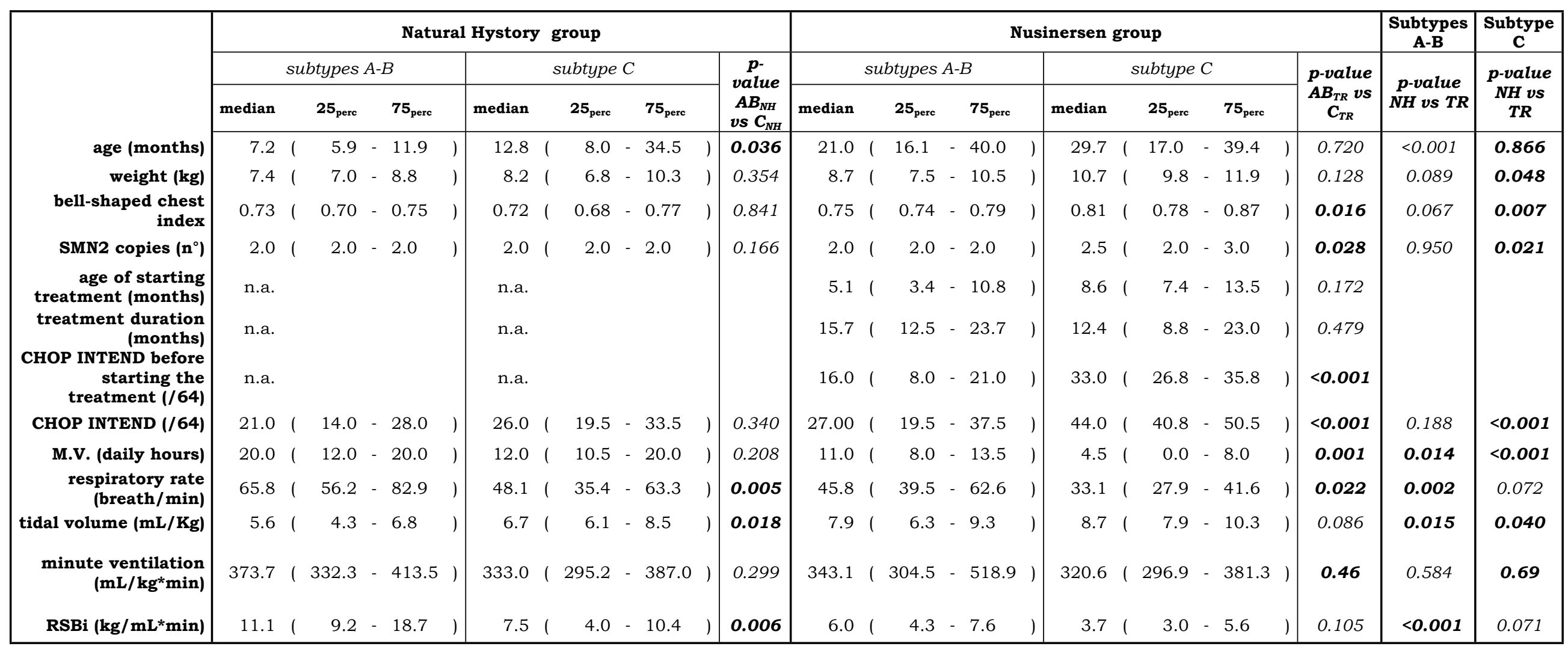

\section{Table 1}

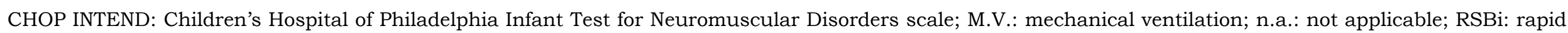

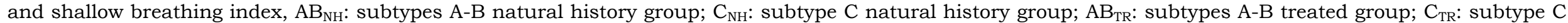
treated group. The computation bell-shaped chest index was previously described(7).

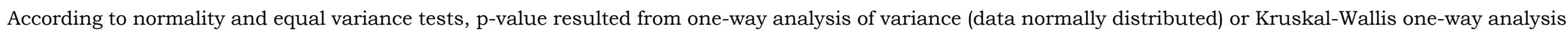
of variance on ranks test (data not normally distributed) with subtypes as independent variables. Significance was set to 0.05 (SigmaStat 3.5, Systat Software, Inc., San Jose, CA, US. 


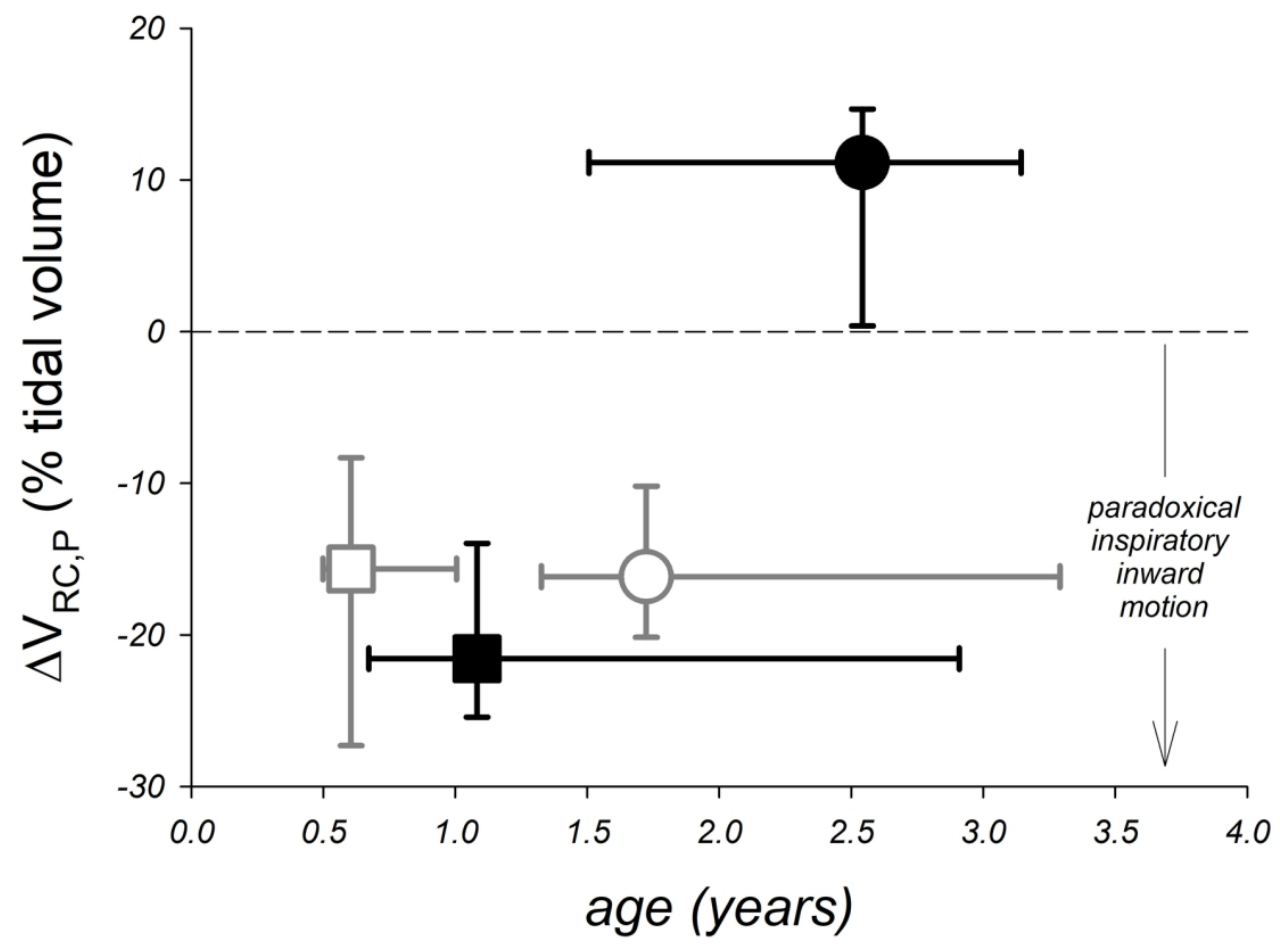

Figure 1

$154 \times 121 \mathrm{~mm}(300 \times 300 \mathrm{DPI})$ 